\title{
The wireless monitoring system of tower cranes based on MCP2515/CAN bus
}

\author{
Yaping Tang, Haibo Wu, Hailong Liu \\ Hunan Railway Professional Technology College, Zhuzhou 412000, China
}

Keywords: MCP2515 driver; Embedded system; CAN bus; Ntelligent montoring.

\begin{abstract}
For the demand of the tower crane with efficient and safe operation, the wireless monitoring management system of a tower crane is designed based on MCP2515/CAN bus in this paper. In order to monitor the operation state information of the tower crane real-time, the system monitor front-end is designed by using the platform with Cortex A8 and WinCE6. MCP2515 driver and Wireless communication message is designed by using Modbus/TCP protocol and the remote monitoring and unified management of the tower cranes are realized by the two-way communication of GPRS and remote management center. The stable and reliable of the system is proved by the field operation and the system has good application prospect.
\end{abstract}

\section{Introduction}

In modern construction is often used tower cranes (referred to as tower crane) carried material handling and loading and unloading operations, due to the tower-altitude operations, complex operating conditions and the operation more difficult, can easily lead to major accidents. In recent years, with the widespread use of the vigorous development of China's economic construction and tower crane, tower crane major accidents caused by increased year by year [1]. In the on-site construction, in order to improve efficiency, it is often illegal tower unit tower crane operations and continuous overload overage use and other issues, greatly increasing the risk of accidents. GB/ T5031-2008 states: lifting machinery certified safety monitoring devices must be installed on the work required to state lifting equipment, rated capacity and risk factors for monitoring and recording, and publishing critical data in real time to a remote monitoring center platform [2], which contains a tower crane. Therefore, the development of stable and efficient wireless tower crane safety monitoring system for security applications crane is important. Important parameters of the system through the sensor will affect the operational safety of the crane, such as the tilt angle, dynamic anti-collision information, hoists, torque, amplitude, height and other information in real-time collection and monitoring, on the one hand through the touch screen display to the field device drivers reference; another aspects of using the Modbus / TCP protocol through GPRS to achieve a seamless link with the remote monitoring center managers to achieve through the Internet for all cranes within the jurisdiction of online monitoring and unified management.

\section{The overall structure and function of a system}

System consists of tower crane monitoring instruments terminal, remote monitoring center and TCP / IP wireless network consists of three parts. After monitoring instruments using ARM Cortex A8 core CPU, main tower machine running real-time sensor data collection to the instrument terminal, GPRS module UART RS485 connection using TCP / IP mode via CAN bus transmission to send data to a remote monitoring center platform; Remote monitoring center is bound with a fixed IP address and port number, sent via Internt GPRS network to receive and manage the monitoring data, and management personnel responsible for sending operational instructions to the appropriate tower crane terminal; TCP / IP wireless network using standard industry MODBUS communication protocol, to ensure data security and stability on the basis of good ductility, very suitable for complex field conditions, the overall structure of the system shown in Fig.1. 


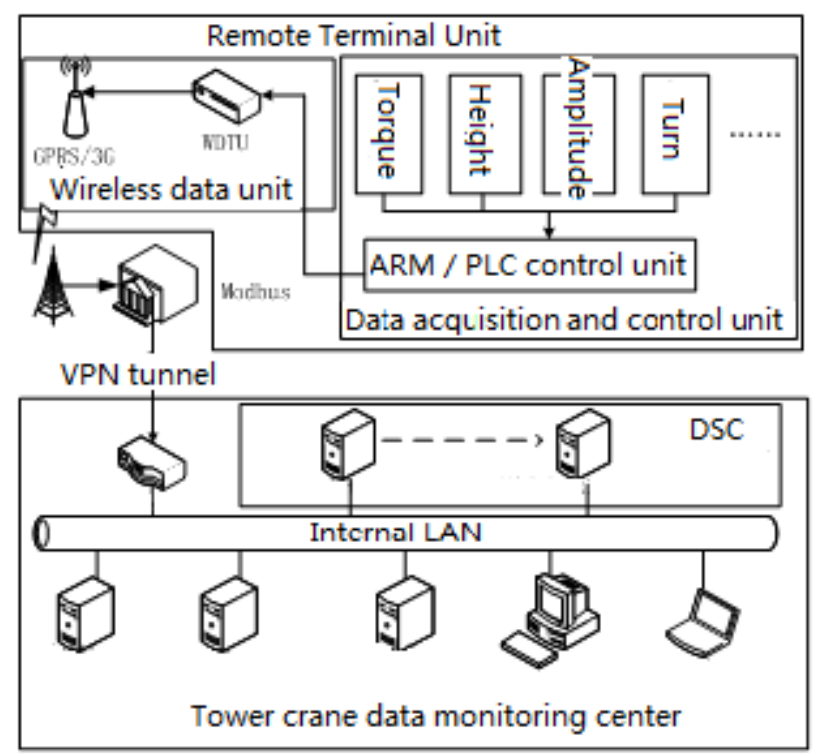

Fig.1 Overall System Architecture

\section{Monitoring terminal hardware design}

Hardware platform using ARM9 Cortex A8 microprocessor core CPU, mainly collected by the sensor network, CAN communication module, alarm control circuit, consisting of 10.4-inch touch screen display, GPRS / GPS / ZigBee wireless communications and other components, the hardware structure of the system shown in Fig.2 .

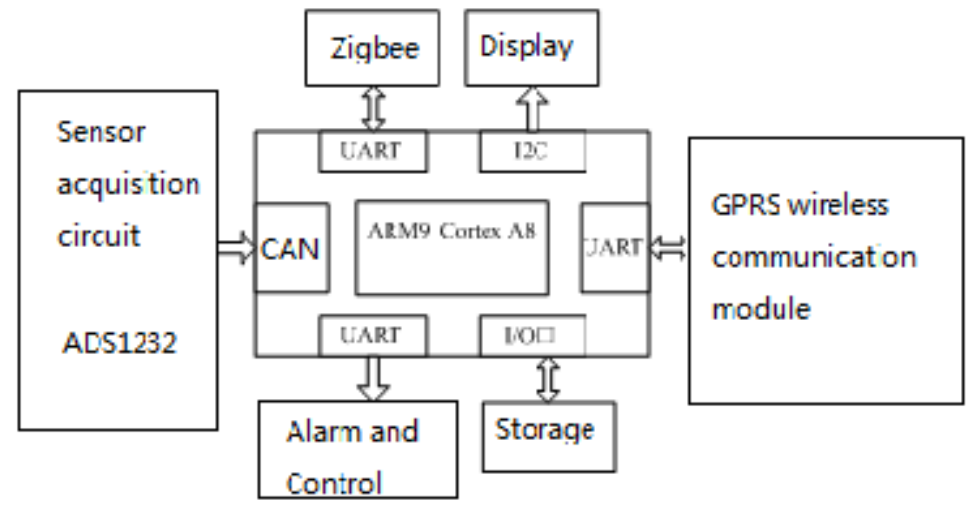

Fig.2 Monitoring terminal hardware architecture

The system uses the latest high-performance ARM9 Cortex A8 microprocessor to control the core, based on S5PV210 core, standard operating frequency is 1G, using single-channel 32bit data bus, 512M DDR2 memory, the perfect support Wince6, Linux and other embedded operating systems [3] . CAN communication module using Microchip's MCP2515 chip, is a stand-alone controller area network protocol controller that supports CAN V2.0B technical specifications, can send and receive standard and extended data frames and remote frames [4]. GPRS module embedded TCP / IP, UDP and other protocols, stable wireless, remote, networked communication, seamless connectivity and Inernet.

\section{MCP2515 Driver Design}

Microchip's MCP2515 is a stand-alone controller area network (Controller Area Network, CAN) protocol controller, which comes with two acceptance masks and six acceptance filters can be realized on the wrong message filtering [5]. WinCE6 system based system design and the preparation of the driver, the device driver, the kernel can not be directly due to identify a physical address, shall be mapped to the physical address space of the virtual address, to determine the physical address 
decoder [6] from the address bus by using MmMapIoSpace () function of the physical and virtual addresses linked to the key code is as follows:

v_pIOPregs= (S3C6410_GPIO_REG*) MmMapIoSpace (ioPhysicalBase, sizeof (S3C6410_ GPIO_REG), FALSE);

Will be conducted after the implementation of the relevant IO MCP2515 initialization mode configurations: standard loopback mode two, the former can not be more than one CAN line for testing; latter is used for standard CAN online communications.

MCP2515_Write (CLKCTRL, MODE_LOOPBACK|CLKEN|CLK8); // Loopback mode

MCP2515_Write (CLKCTRL, MODE_NORMAL|CLKEN|CLK8); // Standard Mode

Followed MCP2515 baud rate settings, CAN bus all nodes must have the same nominal bit rate. Modbus

Protocol uses NRZ ( Non Return to Zero, NRZ) coding. Therefore, the receiving node shall receive clock recovery and synchronization with the transmitter clock. The median time from non-overlapping time segments, using the nominal bit rate (NominalBit Rate, NBR) in the CAN, said it can be used the following equation:

$$
\begin{aligned}
& N B R=f_{b i t}=\frac{1}{t_{b i t}} \\
& t_{b i t}=t_{\text {SyncSeg }}+t_{\text {PropSeg }}+t_{P S 1}+t_{P S 2}
\end{aligned}
$$

In the above formula, the four time periods in turn expressed as a synchronization segment, dissemination section, phase segment PS1, phase segment PS2. In the driver, must meet the PS1> PS2 conditions, the key code is as follows.

MCP2515_Write (CNF1, SJW1|BRP3);//Tbit=20TQ

MCP2515_Write (CNF2, 0xbb);//PS1=8TQ PSeg=4TQ

MCP2515_Write (CNF3, 0x06);//PS2=7TQ SYNC=1TQ

After writing a good drive configuration other load files, add in the project Platform.reg in CAN1.dll, then add the binary image of the code in the project Platform.bib; drive file, create a name for the Makefile, CAN1.def and sources, etc. file for the application layer interface function definition to be output to generate dynamic library specified dynamic library and specify the source file to be compiled, etc., preferably Sysgen command will drive NK.bin compiled into the kernel system in Platform Builder, NK.bin kernel via USB / Jlink programming tools such as the hardware platform to run.

\section{Modbus / TCP protocol-based transmission network.}

Modicon Modbus protocol is developed by an industrial communications protocols and distributed control systems, is a master-slave network, is an application layer packet based transport protocol, the protocol uses a host for one or more slave communication, using the command / answer mode [7]. Where between the host server and the tower crane monitoring terminal via a link TCP / IP way to interact, build the server TCP / IP listener, the terminal is responsible for issuing Kai registration request, after the connection is established by the receiving terminal server reported heartbeat packet to determine the state of a TCP connection . Packet type using Modbus

and Modbus request frame response frame to achieve the command / response packet transmission.

Modbus RTU supports two frame structure and ASC II mode, ASC II mode, each byte packet information unit is divided into two nibbles units, each unit is converted to nibble 7 ASC II character transfer [8] . In the data frame structure, including header, frame length, frame serial number, protocol number, the command character, data payload, CRC checksum and frame tail and other sub-domains, where the data payload type character selected by the command. Communication frame structure of the system control center and tower crane remote monitoring terminal as shown in Tab. 1. 
Tab. 1 Modbus protocol frame structure

\begin{tabular}{lccc}
\hline Header & Frame length & \multicolumn{3}{c}{ Frame serial number } & Agreement No. \\
\hline 2byte & 1byte & 2byte & 1byte \\
\hline $0 \times 5$ A55 & 0x00-0xFF & 0x0000 -0xFFFF & 0x00-0xFF \\
\hline
\end{tabular}

\begin{tabular}{lccr}
\hline Command breaks & Data load & CRC check & Data Frame End \\
\hline 1 byte & $\mathrm{n}$ byte & 1 byte & 2 byte \\
\hline $0 \times 00 \sim 0 \times \mathrm{FF}$ & & $0 \times 00 \sim 0 \times \mathrm{xF}$ & $0 \times 6 A 69$ \\
\hline
\end{tabular}

\section{Field test results}

Based on ARM CortexA8 hardware platform, combined with the touch screen to build friendly interface, to achieve a good impact on the real-time monitoring and recording crane safety data monitoring terminal instrument operating results shown in Figure 4. Through field tests showed that the frequency of large tower crane strong interference conditions, current sensor weight, torque data will appear occasionally jump, malfunction-prone control circuit into account, the system uses the mean filter coefficient select 24 cycles, the test data stable and reliable. Press to write data via RS485 Modbus protocol and GPRS modules for wireless tower crane remote monitoring and management, Internet users can run real-time monitoring and management of the tower crane through the browser interface.

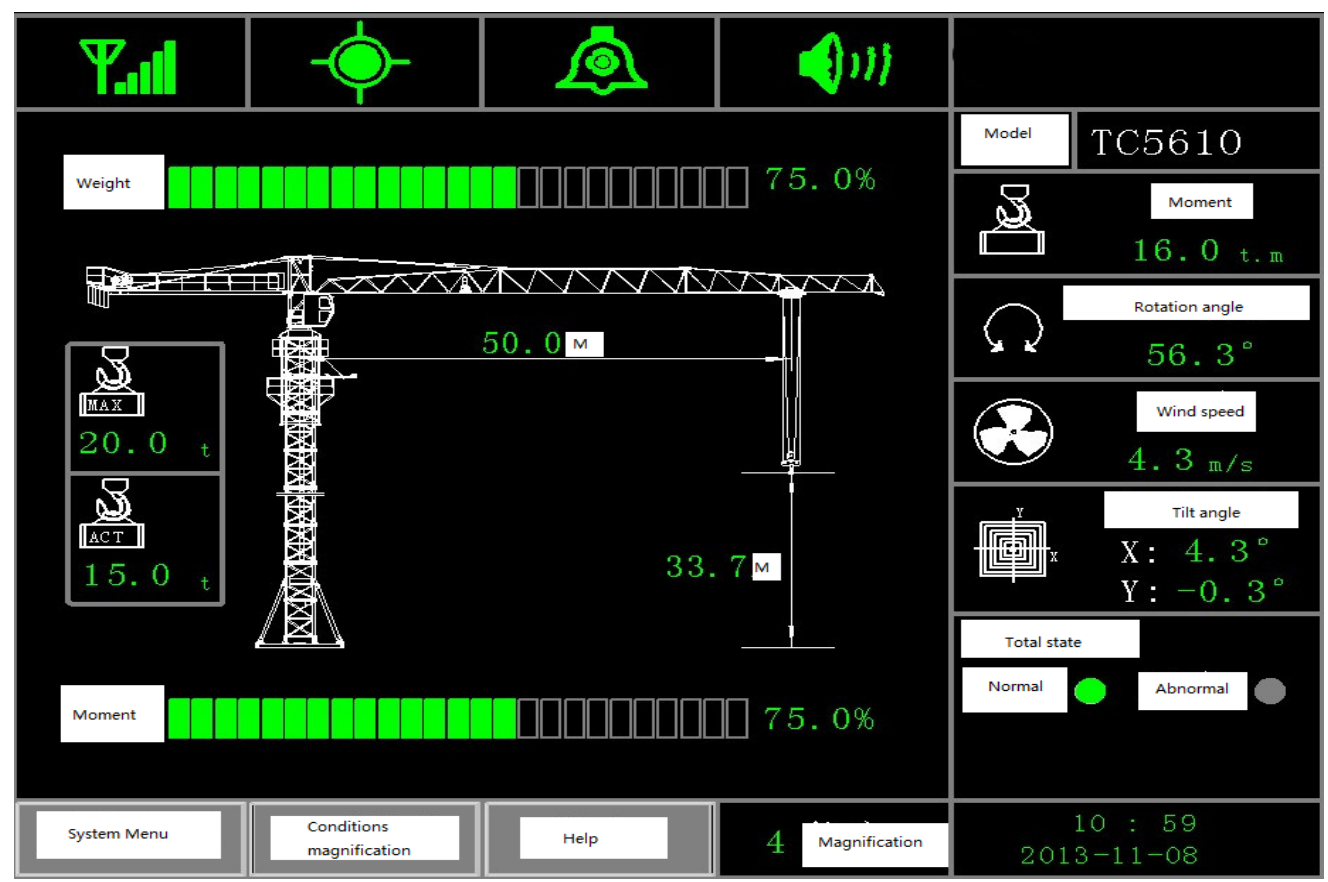

Fig. 4 touch screen interface

\section{Conclusion}

Tower crane wireless remote monitoring and management system for the safe operation of the tower crane and unified management has an important role in most of the domestic like product for the current poor real-time performance of a single, low accuracy, lack of remote monitoring and other issues, is designed based on Modbus / tower crane wireless TCP traffic monitoring and management system, design a stable, efficient, scalable good Modbus communication protocol, developed based on the RS485 device driver wince6 embedded systems design to build a friendly human-machine realization of construction tower site acquisition and local management running the data through GPRS WEB server and build towers to achieve a fleet of remote monitoring and unified management, through on-site commissioning, the system is stable and reliable, and has broad application prospects. 


\section{References}

[1] Wu Xuesong On the Road tower crane safety and sustainable development [J] Construction Mechanization, 2011, Section 01: 21-23.

[2] China National Standardization Committee of tower cranes GB / T 5031-2008 [S] 2008,8: 20-21. [3] Yang Yue, Zheng theoretical, Su Wei, fees keen ACTUATORS industrial wireless network monitoring and control system design and implementation OPC data servers. [J]. Computer Measurement \& Control, 2013,21 (4) 865-870.

[4] Zhou Qicai, Xu Xiaofang, Wang Kai, Xiong Xiaolei, Lu Qiang. Design RS485 bus shield construction ground loss monitoring data acquisition system based on [J] Manufacturing Automation, 2013, (4): 50-55.

[5] Wang Hongwen, Wang Yi Ling, Gao Weiguo. Design and implementation of high-resolution inkjet printer embedded systems [J] Automation Instrumentation, 2013,34 (4): 4: 25-27.

[6] Wang Liping. GPRS-based electric power remote monitoring system [ $\mathrm{J}]$.Communications in Computer and Information Science,2011,86:359 - 364.

[7] Hu Wenxiang, Cai Zheng, Guowei Wei, Wu Yinfeng. Modbus protocol extensions and applications for RS-485 control network [J] Automation Instrumentation, 2013,4 (4): 59-62.

[8] Liu Haibo, Wang Hongyan, Li Aining He Hong. Based on TMS320LF2407A CAN bus data acquisition system [J] Instrument Technique and Sensor, 2014 Section 1: 100-104. 